\title{
Sistemas de tratamento de esgoto sanitário: considerações prévias a avaliação de alternativas e tomada de decisão' ${ }^{1}$
}

\section{Sanitary sewage treatment systems: preliminary considerations for the evaluation of alternatives and decision-making}

\author{
Pedro Bon, Frederico'; Fernandes, Ricardo Augusto Souza 2; Carvalho, \\ Wanessa Karoline Maciel ${ }^{3}$; Queiroz, Andrea Oliveira ${ }^{4}$ \\ 1 Universidade Federal de São Carlos - UFSCar. Departamento de Engenharia \\ Civil - Programa de Pós-graduação em Engenharia Urbana. Rodovia \\ Washington Luís, km 235 - SP-310. São Carlos - São Paulo, CEP 13565-905, Brasil. \\ fredericoejr@gmail.com \\ 2 Universidade Federal de São Carlos, ricardo.asf@gmail.com \\ 3 Universidade Federal de São Carlos, wanessacarvalho29@gmail.com \\ 4 Universidade Federal de São Carlos, andolq@hotmail.com
}

\section{RESUMO}

Os resíduos gerados do uso consuntivo da água afetam negativamente os parâmetros sanitários, estéticos e ecológicos do ambiente natural. Afetam também as populações em termos de saúde e bem-estar. Em épocas em que a responsabilidade com o ambiente natural é crescente e em consonância às restrições legais à proteção e preservação ambiental, a preocupação com a coleta e tratamento de esgotos sanitários é algo imprescindível. Neste contexto, o presente trabalho pretende, por meio da literatura, elencar os fatores técnicos, ambientais, econômicos e sociais, os quais possam ter influência na seleção e tomada de decisão quanto às alternativas de sistemas de tratamento de esgoto. Entende-se que é de altíssima relevância a análise de critérios, visto o déficit de aplicações de ferramentas que combatam as perdas de eficiência e recursos atrelados aos sistemas sanitários. Dessa forma, compreender o setor de saneamento básico e as dificuldades relativas pode colaborar para a determinação das tecnologias que deverão apresentar melhores índices de viabilidade econômica, aceitação, aplicabilidade, implementação, manutenção, operação e eficácia.

Palavras-chave: Estudo de viabilidade, Tratamento de esgoto sanitário, Fatores econômicosociais, Fatores técnicos, Fatores ambientais.

\begin{abstract}
Waste generated from consumptive use of water has negative effects on the sanitary, aesthetic and ecological parameters of the natural environment. They also affect population in terms of health and well-being. In times when the concern for the natural environment is
\end{abstract}

PEDRO BON, Frederico; FERNANDES, Ricardo Augusto Souza; CARVALHO, Wanessa Karoline Maciel; QUEIROZ, Andrea Oliveira. Sistemas de Tratamento de Esgoto Sanitário: Considerações Prévias a Avaliação de Alternativas e Tomada de Decisão. In: II SIMPÓSIO NACIONAL DE GESTÃO E ENGENHARIA URBANA: SINGEURB, 2019, São Paulo. Anais... Porto Alegre: ANTAC, 2019. 
increasing and environmental protection legislation becomes more restrictive, the concern with the collection and treatment of sanitary sewage has grown. The present study represents a theoretical discussion that aims to find in the literature technical, environmental, economic and social factors to be considered when evaluating and choosing alternatives, helping the manager to find the best alternative to his reality. In this context, the present study intends, through the literature, to list the technical, environmental, economic and social factors that may have an influence in the selection and decision making regarding the alternatives of sewage treatment systems. It is understood that the analysis of criteria has extremely importance, since the deficit of tools applications that combat the losses of efficiency and resources tied to the sanitary systems. In this way, understand the basic sanitation sector and its relative difficulties can contribute to the determination of the technologies that shall present better indices of economic viability, acceptance, applicability, implementation, maintenance, operation and effectiveness.

Keywords: Feasibility study, Sanitary sewage treatment, Economic-social factors, Technical factors, Environmental factors.

\section{INTRODUÇÃO}

Os resíduos provenientes das atividades humanas afetam a biota e os parâmetros sanitários e estéticos do meio ambiente, gerando a degradação da qualidade ambiental, que acaba afetando a saúde e o bem-estar da população em geral (BRASIL, 2015).

Segundo a Agência Nacional das Águas (2017), 43\% da população brasileira é atendida por rede coletora e estação de tratamento, $12 \%$ é atendida por solução individual, $18 \%$ é atendida por coleta mas não por afastamento e $27 \%$ desprovida de atendimento. Mesmo assim, sabe-se ainda que $70 \%$ dos 5.570 municípios brasileiros possuem redução de carga orgânica máxima de 30\%, enquanto a resolução CONAMA n 430/2011 estipula 60\% (ANA, 2017). Atualmente, a carga de matéria orgânica total gerada por dia é de pouco mais de 9 mil toneladas, como demonstra a Figura 1.

Figura 1 - Balanço de carga de esgotos

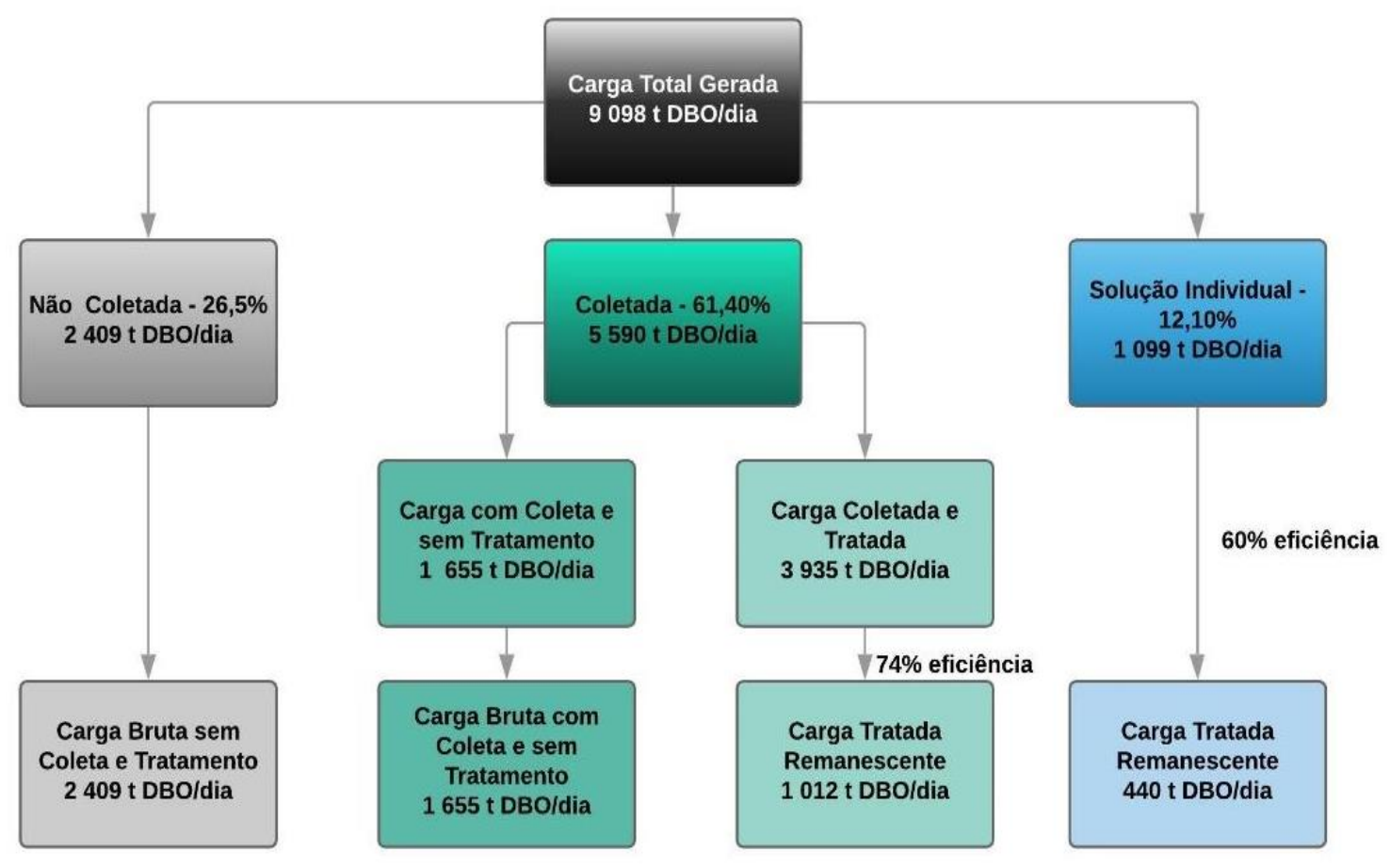

Fonte: Adaptada de Agência Nacional de Águas (2017)

Dos 1.587 municípios que declaram tratar o esgoto (BRASIL, 2015), alguns aplicam 
simplesmente métodos de gradeamento. Tais ações promovem a separação dos resíduos físicos que muitas vezes são inertes, mas não minimizam seu potencial de alterar química e biologicamente os corpos receptores.

Porém, o baixo índice de atendimento por rede pública de esgotamento não deveria estar associado a más condições sanitárias, visto que em muitos países as residências são providas de soluções individuais ou descentralizadas e que promovem disposição final adequada (BRASIL, 2015). Para que esse panorama se instale no Brasil, é preciso que os cidadãos sejam instruídos sobre a importância de construir e dar manutenção às instalações de tratamento de efluentes sanitários.

A chamada evolução dos sistemas de coleta de esgoto no Brasil, resultou em uma população que privilegia o afastamento do efluente das residências, sem preocupação com sua destinação e análise dos impactos que causam (MARTINETTI; SHIMBO; TEIXEIRA, 2007).

Este estudo representa uma discussão teórica que objetiva encontrar na literatura quais são os fatores técnicos, ambientais, econômicos e sociais a serem considerados no momento de avaliação das alternativas e instalação de um sistema de tratamento de esgotos, auxiliando o gestor a fazer considerações acuradas com a sua realidade no momento da escolha.

\section{METODOLOGIA}

Os principais materiais utilizados corresponderam a obras da literatura, com base nas quais foram levantados os dados de caracterização das alternativas tecnológicas e locacionais para o tratamento de esgotos sanitários. Assim, foi traçado um esquema metodológico de análise bibliográfica dividido em três categorias principais, resultando em um levantamento, tanto a nível nacional como internacional, referente aos critérios avaliados para a tomada de decisão e suas possíveis aplicações na escolha de sistemas de tratamento de esgoto sanitário.

\section{RESULTADOS E DISCUSSÃO}

\subsection{Importância da caracterização prévia das alternativas na implantação de um sistema de tratamento de esgotos sanitários}

O processo de escolha de alternativas tecnológicas e locacionais envolve múltiplas interfaces devendo ser construída em consonância aos aspectos técnicos, econômico-financeiros, sociais, ambientais, legais e inclusive os políticos (CAMPOS, 2011), como sinoticamente ilustrado pela Figura 2.

Figura 2 - Fatores que determinam a melhor alternativa

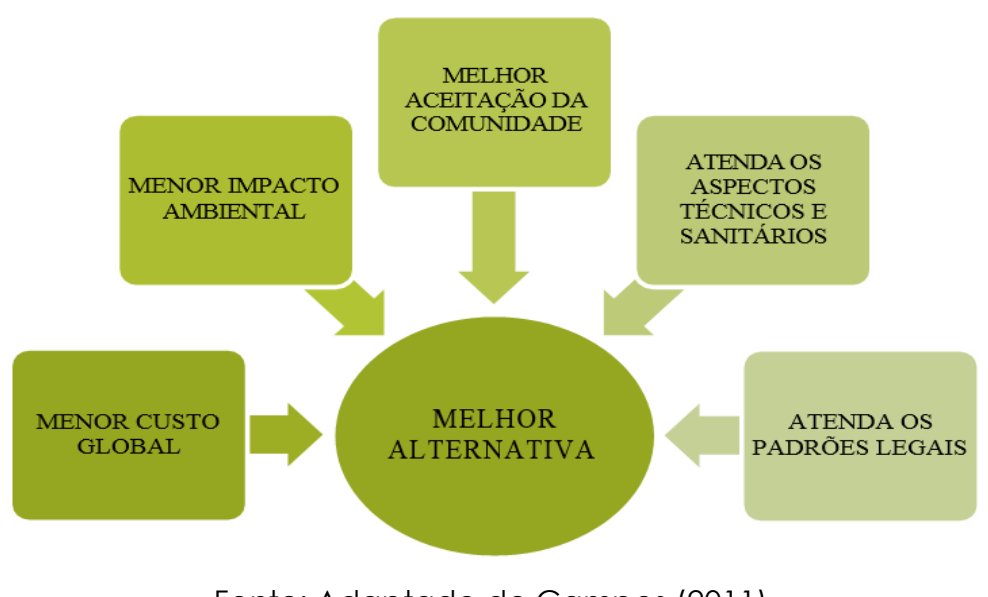

Fonte: Adaptado de Campos (2011)

Compreender a dimensão que abarca as alternativas para tratamento de esgotos sanitários 
é fundamental ao processo de escolha, visto que conhecer as tecnologias disponíveis e as condicionantes locais é o ponto chave para iniciar um estudo de viabilidade.

Diante da escassez de recursos, a escolha da melhor alternativa será a que atenda às legislações vigentes, considerando os impactos ambientais e que apresente baixos custos de implantação, manutenção e operação, sem excluir o atendimento de outros critérios (ZANIN, 2011).

A garantia da qualidade da decisão está na adequada definição dos critérios de avaliação (CAMPOS, 2011). Desta perspectiva, pretende-se compreender os fatores envolvidos na definição das alternativas tecnológicas e locacionais para tratamento de esgoto sanitário. $O$ Quadro 1 elenca os aspectos e critérios segundo as dimensões técnica, econômica, ambiental e social.

Quadro 1 - Aspectos e critérios relacionados às alternativas tecnológicas e locacionais para ETE

\begin{tabular}{|c|c|c|}
\hline Aspectos & & Critérios \\
\hline \multirow{23}{*}{ Técnico } & \multirow{2}{*}{ Demanda } & Nível de tratamento requerido \\
\hline & & População atendida \\
\hline & \multirow{7}{*}{ Físicos } & Geologia \\
\hline & & Geomorfologia \\
\hline & & Pedologia \\
\hline & & Hidrografia \\
\hline & & Fatores hidrológicos \\
\hline & & Qualidade do corpo receptor \\
\hline & & Clima \\
\hline & \multirow{3}{*}{ Biótico } & Clima \\
\hline & & Vegetação \\
\hline & & Fauna \\
\hline & \multirow{5}{*}{ Antrópico } & Estrutura fundiária \\
\hline & & Uso e ocupação do solo \\
\hline & & Infraestrutura \\
\hline & & Valor da terra/desapropriações \\
\hline & & Recursos humanos e tecnológicos \\
\hline & \multicolumn{2}{|c|}{$\begin{array}{l}\text { Jurídicos e institucionais } \\
\text { Aplicabilidade/ maturidade da tecnologia } \\
\text { Confiabilidade } \\
\text { Complexidade/ gerenciabilidade/simplicidade operacional e manutenção } \\
\text { Riscos } \\
\text { Durabilidade } \\
\text { Robustez/ flexibilidade do sistema }\end{array}$} \\
\hline & \multirow{5}{*}{ Desempenho/eficiência } & Remoção DQO \\
\hline & & Remoção DBO \\
\hline & & Remoção de sólidos \\
\hline & & Remoção de nitrogênio \\
\hline & & Remoção de fósforo/ eutrofização potencial \\
\hline
\end{tabular}




\begin{tabular}{|c|c|c|}
\hline & & $\begin{array}{l}\text { Remoção de Patógenos/ patogenicidade e geração de } \\
\text { subprodutos }\end{array}$ \\
\hline & & Produção/geração de lodo \\
\hline & & Limitações do tratamento \\
\hline & \multicolumn{2}{|c|}{ Potencial para recuperar produtos/ potencial de reuso } \\
\hline Econômico & \multicolumn{2}{|c|}{$\begin{array}{l}\text { Custo de implantação e investimentos } \\
\text { Custos de operação e manutenção } \\
\text { Requisitos de produtos químicos } \\
\text { Requisitos energéticos e consumo de energia } \\
\text { Área requerida e requisitos da área } \\
\text { Custos de disposição do lodo } \\
\text { Processos auxiliares requeridos } \\
\text { Custos do ciclo de vida }\end{array}$} \\
\hline \multirow{13}{*}{ Ambiental } & \multirow{4}{*}{ Atmosfera } & Alteração da qualidade do ar (odores) \\
\hline & & Emissões e aquecimento global potencial \\
\hline & & Ruídos \\
\hline & & Aerossóis \\
\hline & \multirow{3}{*}{ Água } & Alteração da qualidade das águas superficiais e subterrâneas \\
\hline & & Assoreamento dos corpos d'água \\
\hline & & Eutrofização \\
\hline & Solo & Alteração da qualidade dos solos \\
\hline & \multirow{2}{*}{ Físico } & Uso/ modificação de espaço físico \\
\hline & & Impacto visual/ estética \\
\hline & \multirow{2}{*}{ Biota } & Danos a flora e a fauna \\
\hline & & Alteração de insetos \\
\hline & Sanitária & Riscos sanitários \\
\hline Social & \multicolumn{2}{|c|}{$\begin{array}{l}\text { Custos para os usuários } \\
\text { Condições de vida da população } \\
\text { Aspectos culturais e da organização social/ política } \\
\text { Aceitação pública e aceitabilidade } \\
\text { Geração de empregos }\end{array}$} \\
\hline
\end{tabular}

Fonte: Adaptado de GOFFI (2017), LINS (2011) e SANTOS (2004)

Assim, Zanin (2011) entende que os métodos multicritérios são capazes de agregar todas as características importantes na análise, permitindo um processo transparente e sistematizado quanto aos problemas de tomada de decisão.

\subsection{Ferramentas para análise e escolha de alternativas sustentáveis de tratamento de esgotos sanitários}

Atualmente, o emprego do saneamento implica na superação de entraves políticos, tecnológicos e gerenciais (GUIMARÃES; CARVALHO; SILVA, 2007) e o que se busca são formas alternativas que combinem baixo custo (variável econômica) com eficiência (variável técnica-ambiental), alinhadas com as necessidades dos usuários (variável social) (CORNELLI, 2014). 
A maioria dos gestores têm necessidade de consultoria técnica para tomada de decisões que exigem conhecimento prévio quando o assunto é saneamento, uma vez que os objetivos e os critérios muitas vezes disputam entre si. As ferramentas, portanto, os auxiliam na seleção de métodos mais sustentáveis (CORNELLI, 2014) para que o aspecto econômico não seja o único levado em consideração.

Cornelli (2014) levanta critérios que devem ser considerados no momento de escolher uma alternativa, como: i) a identificação de alternativas de tratamento ambientalmente viáveis; ii) a identificação de alternativas tecnicamente viáveis e que atendam às legislações restritivas; iii) as demandas atuais e futuras para a vida útil do projeto; e iv) o desenvolvimento de uma ferramenta com critérios de seleção de alternativas. Hunt (2013) realizou um levanto bibliográfico sobre os quesitos a serem atendidos por um sistema de tratamento de esgotos. Cada solução (coletiva ou individual), cada localidade, cada população e cada gestão apresentavam diferentes necessidades técnicas, sociais, ambientais e econômicas para o mesmo problema do tratamento.

As necessidades mais latentes identificadas foram: nível do tratamento necessário, possibilidade de reuso, qualidade da água do corpo receptor, confiabilidade da alternativa, compatibilidade, flexibilidade, investimento para implantação, dificuldade de construção, dificuldade de ampliação, custo de operação e manutenção, demanda de área, independência do solo, localização da estação em relação ao ponto de descarga, altitude, necessidade de sistemas elevatórios, declividade, impacto urbanístico, impacto arquitetônico, acessibilidade, distância em relação ao perímetro urbano, ruídos, tempo de detenção hidráulica, demanda de energia elétrica, quantidade de lodo produzida, simplicidade operacional, impacto ambiental, aceitação pública, estética, geração de odor, geração de ruídos, alterações no clima, zoneamento, possibilidade de problemas ambientais, possibilidade de atração de insetos e vermes.

Das 18 variáveis avaliadas por Hunt (2013), a variável econômica foi priorizada. Este critério é importante, tendo em vista a limitação de recursos financeiros das prefeituras, mas a médio e longo prazo uma decisão calcada em fatores econômicos pode resultar em defasagem de fatores ambientais e sociais muito mais onerosos, principalmente se acarretar recuperação de áreas danificadas (CORNELLI, 2014). O desenvolvimento de uma ferramenta que preza pela sustentabilidade, pode ser dividido em três etapas conforme a demonstrado pela Figura 4, que segue dividida em 3 colunas, as quais respectivamente, apresentam: (1) as etapas; (2) as atividades a serem desenvolvidas e; (3) os tópicos sobre os quais cada etapa permite obter conclusões. segue organizada.

Figura 4 - Etapas de desenvolvimento de uma ferramenta de seleção de alternativas no tratamento de esgoto

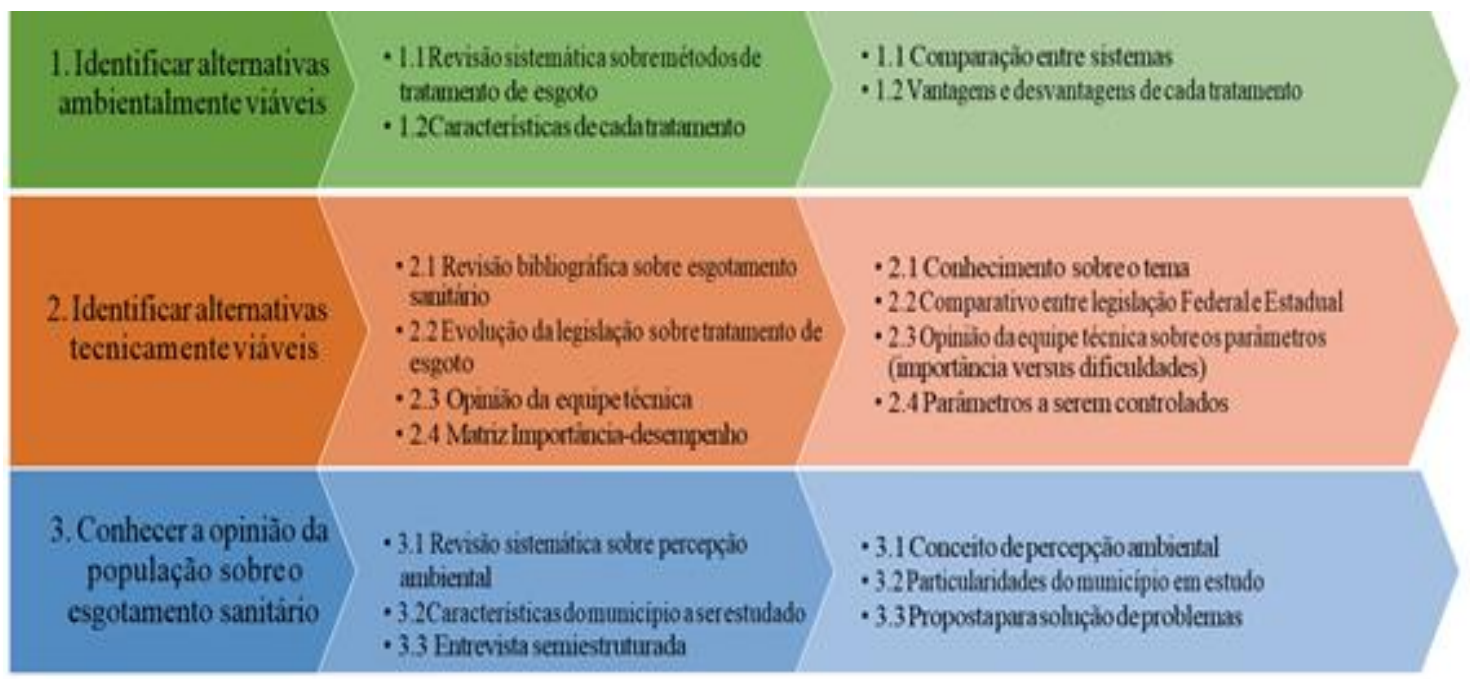

Fonte: Cornelli (2014) 
Dito isto, Cornelli (2014) explica sua metodologia da seguinte maneira:

- Etapa 1 permite identificar as alternativas mais passíveis de uso segundo o aspecto ambiental;

- Etapa 2 propõe conhecer a legislação aplicável na localidade além de atender a resolução CONAMA n 357/2005 e resolução CONSEMA n 128/2006;

- Etapa 3 pretende levar o gestor a compreender a percepção da população sobre as alternativas de tratamento de esgotos que estão sendo cotadas para aquela localidade, a partir de entrevistas.

Assim, percorrendo as etapas e aprofundando-se uma a uma, o gestor consegue atribuir pesos a cada critério. Cabe ressaltar que os critérios aos quais se atribuem os maiores pesos por parte de uma população, um governo local e uma situação econômica-ambiental nunca serão os mesmos em outra localidade, de modo que cada alternativa deva ser adequada aos seus usuários e ao seu ambiente de inserção e, portanto,, a alternativa escolhida nunca deverá ser genérica, mas adaptável ao contexto envolvido.

\section{CONCLUSÕES}

Considerando a importância do tratamento dos esgotos sanitários no Brasil, têm-se estudado sobre o desenvolvimento e avaliação de diferentes tecnologias e processos de tratamento deste tipo de resíduos. No entanto, ainda é pouco explorada a área de estudos sobre o processo de tomada de decisão e as metodologias que podem determinar a tecnologia que deverá ser empregada em um certo local, com o intuito de garantir a máxima eficiência na definição do sistema de tratamento. Neste sentido, conclui-se que é de altíssima relevância a análise de critérios que auxiliarão de forma efetiva na etapa de planejamento e escolha da melhor tecnologia a ser utilizada em determinado local, haja visto o déficit de aplicações de ferramentas que resultem na tomada de decisões mantendo a qualidade dos serviços e combatendo as perdas de eficiência e recursos atrelados aos sistemas sanitários. Desta forma, este trabalho contribui para compreender o setor de saneamento básico e as dificuldades relativas à determinação do sistema de tratamento para cada situação, devido, principalmente, à falta de informações e direções para a tomada desta importante decisão. Buscou-se entender quais aspectos devem ser considerados na determinação das tecnologias que, ao final, podem apresentar os melhores índices de viabilidade econômica, boa aceitação, aplicabilidade, simplicidade de implementação, manutenção e operação e eficácia nos resultados obtidos.

\section{AGRADECIMENTOS}

O presente trabalho foi realizado com apoio da Coordenação de Aperfeiçoamento de Pessoal de Nível Superior - Brasil (CAPES) - Código de Financiamento 001 e também da Fundação de Amparo à Pesquisa ao Desenvolvimento Científico e Tecnológico (FAPEMA) Processo BM-08469/17.

\section{REFERÊNCIAS}

\section{ANA - AGÊNCIA NACIONAL DE ÁGUAS (Brasil). Atlas esgotos: despoluição de bacias} hidrográficas. Agência Nacional de Águas, Secretaria Nacional de Saneamento Ambiental. Brasilia: 2017.

BRASIL. Ministério da Saúde. Fundação Nacional da Saúde (FUNASA). Manual de Saneamento. 4. ed. Brasília. 2015. 642 p.

CAMPOLINA, A. G. et al. Análise de decisão multicritério para alocação de recursos e avaliação de tecnologias em saúde: tão longe e tão perto? Cadernos de Saúde Pública, v. 33, n. 10, pp.1-15. 2017 
CAMPOS, V. R. Modelo de apoio à decisão multicritério para a priorização de projetos em saneamento. Tese (Doutorado - Programa de Pós-graduação em Engenharia de Produção). Escola de Engenharia de São Carlos da Universidade de São Paulo. São Carlos. 175p. 2011.

CORNELLI, R. Análise e Seleção de Alternativas Sustentáveis de Esgotamento Sanitário. 2014. 136 f. Tese (Doutorado) - Curso de Engenharia de Produção, Programa de Pósgraduação em Engenharia de Produção, Universidade Federal do Rio Grande do Sul, Porto Alegre, 2014.

GOFFI, A. S. Uso da análise multicritério para seleção de tecnologias de tratamento de efluentes. Dissertação (Mestrado - Programa de Pós-graduação em Engenharia de Produção e Sistemas). Universidade Tecnológica Federal do Paraná. Pato Branco. 113p. 2017.

GUIMARÃES, A. J. A.; CARVALHO, D. F. de; SILVA, L. D. B. da. Saneamento básico. Disponível em: http://www.ufrr.br/institutos/it/deng/leonardo/downloads/APOSTILA01.pdf Acesso em: 20 ago. 2018.

HUNT, C. C. Modelo multicritério de apoio à decisão aplicado à seleção de sistema de tratamento de esgoto para pequenos municípios. 2013. 118p. Dissertação (Mestrado em Engenharia Ambiental), Programa de Engenharia Ambiental, Escola Politécnica e Escola de Química, Universidade Federal do Rio de Janeiro, Rio de Janeiro, 2013.LINS, G. A. Impactos ambientais em estações de tratamento de esgotos (ETEs). Dissertação (Mestrado profissional - Programa de Pós-Graduação em Engenharia Ambiental). Universidade Federal do Rio de Janeiro. Rio de Janeiro. 285p. 2010

MARTINETTI, T. H.; SHIMBO, I.; TEIXEIRA, B. A. N. Análise de Alternativas mais Sustentáveis para Tratamento Local de Efluentes Sanitários Residenciais. In: IV ENCONTRO NACIONAL E II ENCONTRO LATINO AMERICANO SOBRE EDIFICAÇÕES E COMUNIDADE SUSTENTÁVEIS, 2007 , Curitiba. Anais. Curitiba: S.I., 2007. p. 996 - 1005. Disponível em:

http://www.elecs2013.ufpr.br/wp-content/uploads/2007_artigo_019.pdf. Acesso em: 29 ago. 2018.

RUSCASSIER-CHADIRAT, G. \& DEUTSCH, J-CL. Development and implementation of sewerage programmes: proposal of a methodology for the decision process. Proc. 2nd Int. Conf. on Decision Making in Urban and Civil Engineering, Lyon, France, pp. 639-642. 2000

SANTOS, R. F. D. Planejamento Ambiental: teoria e prática. São Paulo: Oficina de Textos, 2004.

TCHEMRA, A. H. Tabela de decisão adaptativa na tomada de decisões multicritério. Tese (doutorado). Escola politécnica da Universidade de São Paulo. Departamento de engenharia de computação e sistemas digitais. São Paulo. 172 p. 2009

THOKALA, P. et al. Multiple criteria decision analysis for health care decision making - an introduction. Report 1 of the ISPOR MCDA Emerging Good Practices Task Force. Value Health; pp. 1-13. 2016

ZANIN, R. A. Seleção de processos de tratamento de esgoto sanitário utilizando análise multicritério. Dissertação (Mestrado - Engenharia de Processos). Universidade da Região de Joinville. Joinville: UNIVILLE. 121p. 2011. 